\title{
Distinctions Between Central and Peripheral Hearing
}

\author{
Ahmad Yousef ${ }^{1}$ \\ 'School of Computational Science and Engineering, McMaster University, Hamilton, Ontario, Canada \\ *Correspondence: mohamas2@mcmaster.ca
}

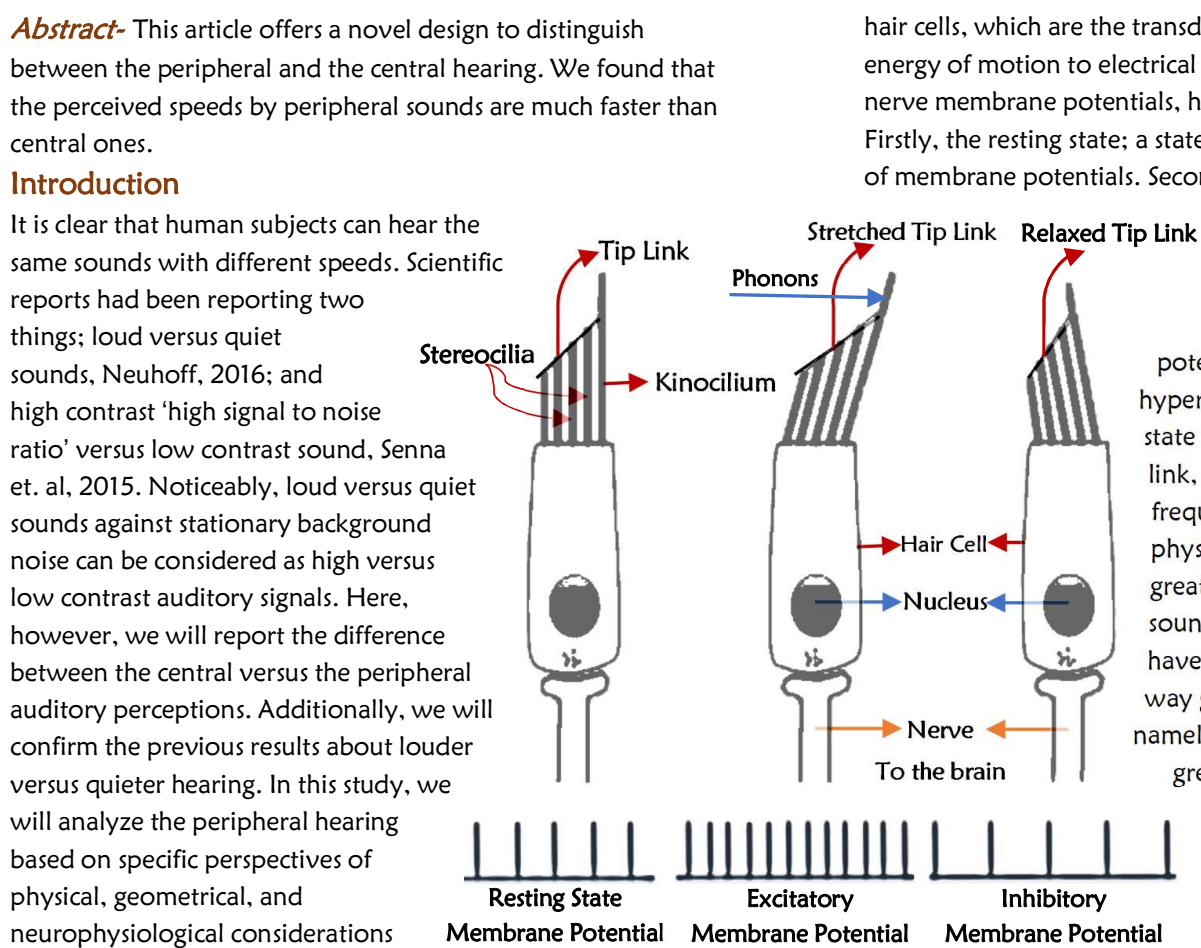
that should be in harmony with

the common definition of the peripheral vision. In vision research, objects in the peripheral vision are seen smaller in size (increase in spatial frequency) and faster in speed, see reference 4. The recent retinal 'physiological and anatomical' findings may agree with the aforementioned psychophysical report, see reference 5 , and 6 . In physics, phonons are considered as quasiparticles associated with compressional sound waves. We therefore define the central hearing as a conscious process produced by straight phonons' rays inside the ear

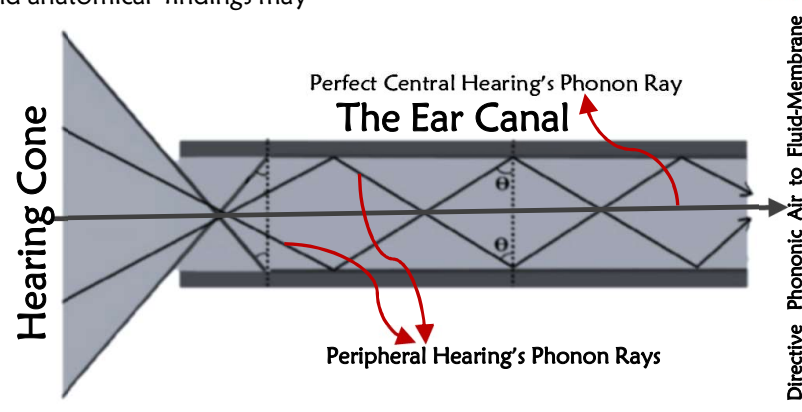
Poreeds when the sounds are heard centrally. Notice that, in our design, the phonon rays for central condition move mostly in straight direction inside the ear canal; namely it will subtend a smaller 'auditory angle'; a complex analogical term of the visual angle potentials than lower sounds. That neurophysiological reason could be the main reason behind why the humans had been consciously hearing various speeds frequency of membrane potentials. Thirdly, the yperpolarization state; an inhibitory where the hair cell has relaxed tip frequencies of membrane potentials. In physics, louder sounds should carry number of phonons than quiet have the ability to stretch the tip link way greater than quieter sounds, mely, louder sounds should produce greater frequency of membrane which will be discussed in a further study. However, for the peripheral hearing 은 straight in the ear canal, instead they have 'zigzag' pathways, thus the phonon rays will have the ability to subtend greater 'auditory angle'. Detailed canal towards the inner ear. Peripheral hearing, however, have snaky phonons' rays due to its peripheral presentation. To approach the greatest peripheral presentation, and to ensure that distance between the speaker and the left ear is equal to the speaker and the right ear, we placed the headphone centrally away and in front of the face of the human subject. In previous studies, we had theorized that the human visual system has two conscious brains, namely, a central conscious brain, and a peripheral one. Similar to the human visual system, the current work aims to provide virtuous evidence that the human auditory system also has two conscious brains. Before presenting the results of our study, we have to understand the possible neurophysiological reasons behind hearing fast versus slow speed of the same soundtrack. As shown in the central infographic, the neurophysiological explanation of the peripheral hearing condition will be offered in the discussion section.

Materials \& Methods

Twelve human subjects, with dilated pupils, were recruited to participate in this study. Each human subject has to hear two different soundtracks of an original recording of train sound; see reference 7. We use terminologies 'central hearing' for 'headphone on the ears' and 'peripheral hearing' for 'headphone off the head (on the table in front of the human subjects, namely, 40 centimeters away from them)'. Since we are not sure whether the ears of human subjects have different auditory thresholds; we tested the right ear and the left ear in sperate sessions. Namely, human subjects will have 'Left :ON; Right: OFF' trails and then the opposite operation for counterbalancing. The first soundtrack 
is for the central hearing sessions, see reference 7. These sessions are to compare the frame of reference "high pressure, central hearing; normalized value of 1 " with "low pressure, central hearing; normalized value of $1 / 3$. The second soundtrack is for the peripheral hearing sessions, and it is to compare the frame of reference 'high pressure, central hearing; normalized value of 1' versus low pressure peripheral hearing; normalized value of $1 / 3$. Important to emphasize, the frame of reference for the central and peripheral sessions is the same, namely, it's central hearing (headphone on the ears) with normalized value of 1.

Importantly, for statistical analysis of the peripheral hearing sessions, the headphone should be precisely placed 40 centimeters away and in front of the human subject for the peripheral hearing condition to guarantee normalized value of $1 / 3$, for better details see reference 7 . Namely, the normalized value will be changed from 1 to $1 / 3$ due to the change in the spatial position of the headphone, because it's removed from the head, and the placed in the front of human subjects, which should result in significant reduction of the normalized pressure as previously mentioned.

\section{Data Recording}

The data are collected manually after each session, namely, the human subjects completed four sessions, two for 'central hearing' test (right or left ear), and two for 'peripheral hearing' test. The outcome of the two central hearing tests are then averaged for each human subject; and the same operation was applied to the peripheral hearing tests. The resultant data afterwards are used to obtain the simplest statistical values, namely, the average and the standard error of the mean for the twelve subjects.

Results and Discussion

As shown in the infographic, peripheral hearing appears to be perceived significantly faster than the central hearing, despite that fact that the central hearing has three times higher pressure than the peripheral hearing. The

aforementioned finding does not invalidate the previous scientific findings, namely, references 1,2 , and our reproduction (the lefthand side of the central figure); however, it emphasizes that we must distinguish between central and peripheral hearings. The neurophysiological reason behind this finding could be because the peripheral hearing might allow greater number of phonons to subtend larger 'auditory angle' in the inner ear. Namely, that larger 'auditory angle' might motivates a lot of 'temporally asynchronized' hair cells to be activated. Temporally asynchronized hair cells refer to not only whether different cells are able to signal the conscious brain in different times; but also, whether different cells are able to produce different membrane potentials for similar amounts of interactive phonons. Based on our tests, these temporal asynchronies had been always leading to wavelength constriction at the level of auditory awareness. Importantly, similar to the temporal differences of the signals produced by the retinal cells; the hair cells in the peripheral areas of the inner hair might be signaling the brain much faster than those hair cells in the ear's macula; which adds further neurophysiological explanation to our finding. Summarily, when the sound was centrally presented, the perceived speed was slow, thus larger wavelength; but, when it was peripherally displayed, the perceived speed was much faster, thus shorter wavelength. This finding is astoundingly consisted with the theory of two conscious brains, see reference 4 , and 8 . The offered explanations might not be able to speculatively discard possibilities of 'quantum hearing'; namely, the phonons produced in peripheral or central auditory spaces might be quantumly entangled with their corresponding hair cells in corresponding areas resided in the inner ear. Whether the 'quantum hearing' speculation is true; the conventional physical and neurophysiological explanations seem to provide sufficient scientific reasoning.

Out of the scope, peripheral vision might be geometrically coupled with central hearing. Integratively speaking, allocation of cognitive resources to hearing 'auditory attention', had been often triggering pupil dilation for greater access to the peripheral vision, see reference 9 . We therefore speculate that 'auditory attention' intensifies the peripheral vision. In another word, 'auditory attention' might construct a novel audiovisual contrast sensitivity function, resembles to the visual contrast sensitivity function, see reference 10; in order to acquire more comprehensive audiovisual integration, see reference 11.

\section{Essential Notification}

Readers should have direct access to the stimuli, and to the instructions that control each of the two speakers (e.g, mute the right speaker, and keep the left), for download, see reference 7 . For rapid validation process, it is must for the readers to have
Frame of Reference accessibility to a headphone and should have dilated pupils. For the best results, kindly do not try the experiment with pinpoint pupils. Readers may try the stimuli with both of the headphone's speakers $\mathrm{ON}$; it will produce similar effect, in terms of speed, but the volume will be heard little quieter. The readers should connect the author directly in case of they cannot access the stimuli or unable to comprehend the instructions. Scholars with brain imaging facilitations are

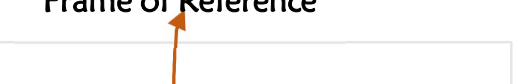

welcome to collaborate in the aforementioned challenging investigations. Enquiries should be sent to the author.

\section{Transactional References}

[1] J, Neuhoff, 2016. Looming sounds are perceived as faster than receding sounds. Cogn Res Princ Implic. 2016.

[2] I. Senna, etal. 2015. Hearing in slow-motion: Humans underestimate the speed of moving sounds. Scientific Reports 5.

[3] Kelley, etal. (2005). Development of the Inner Ear. Springer.

[4] Yousef, Ahmad. 2019. "Spatial Attention Dilates Time and Length." PsyArXiv. doi:10.31234/osf.io/qazj9.

[5] Sinha, etal. (2017). Cellular and circuit mechanisms shaping the perceptual properties of the primate fovea. Cell 168, 413-426. [6] Masland, (2017). Vision: Two Speeds in the Retina. Current Biology. [7] Stimuli and instructions may be found in the following link: https://drive.google.com/drive/folders/1mV7H7WHCgyuEskBnyjGBOd3Aj Y6IGcld?usp=sharing

[8] Yousef, A. 2019. "Two Distinct Fusiform Face Areas." PsyArXiv. doi:10.31234/osf.io/a8gzv.

[9] Zekveld, etal. (2018)." The Pupil Dilation Response to Auditory Stimuli: Current State of Knowledge." Trends in Hearing, 2018.

[10] Campbell, and Robson (1967). Application of fourier analysis to the visibility of gratings. J. Physiol. (1968), 197, pp. 551-566.

[11] Noel, etal. (2018). Audiovisual integration in depth: multisensory binding and gain as a function of distance. Exp Brain Res. 2018. 